\title{
GENERATION OF MAGNETIC FIELDS IN COSMOLOGICAL SHOCKS
}

\author{
Mikhail V. Medvedev ${ }^{1,2}$, Luis O. Silva ${ }^{3}$, Massimiliano Fiore $^{3}$, Ricardo A. Fonseca ${ }^{3}$, And Warren B. Mori ${ }^{4}$ \\ ${ }^{1}$ Department of Physics and Astronomy, University of Kansas, KS 66045, USA \\ ${ }^{2}$ Institute for Nuclear Fusion, RRC "Kurchatov Institute", Moscow 123182, Russia \\ ${ }^{3}$ GoLP/Centro de Fisica de Plasmas, Instituto Superior Técnico, 1049-001 Lisboa, Portugal \\ ${ }^{4}$ Department of Physics and Astronomy, University of California, Los Angeles, CA 90095, USA \\ E-mail: medvedev@mail.ku.edu
}

\begin{abstract}
The origin of magnetic fields in the universe remains an outstanding problem in cosmology. We propose that these fields are produced by shocks during the large-scale structure formation. We discuss the mechanism of the field generation via the counter-streaming (Weibel) instability. We also show that these Weibel-generated fields are long-lived and weakly coupled to dissipation. Subsequent field amplification by the intra-cluster turbulence may also take place, thus maintaining the magnetic energy density close to equipartition.
\end{abstract}

Key words : cosmology: large scale structure - magnetic fields - shock waves

\section{INTRODUCTION}

The existence of (sub-) micro-gauss magnetic fields in galaxy clusters is now a well-established fact. Multiple observations indicate their presence by Faraday rotation and other observables. However, the question of the origin of these fields remains open. Traditionally, one considers the amplification of seed fields by hydrodynamic turbulence excited during violent processes of large-scale structure formation. The seed fields, in turn, may be of primordial origin or be produced locally. In the former case, they could have been produced during anQCD or electroweak first order phase transition (Quashnock, Loeb, \& Spergel, 1989; Vachaspati, 1991; Sigl, Olinto, \& Jedamnik, 1997), or during (dilaton-driven) inflation (Ratra, 1992; Gasperini, Giovannini, \& Veneziano, 1995). The non-primordial models include the Harrison mechanism (Harrison, 1970; Sicotte, 1997), Biermann battery (Biermann, 1950; Gnedin, Ferrara, \& Zweibel, 2000), the field generation from primordial helicity (Field \& Carroll, 2000; Sigl, 2001), the field generation in collisionless shocks (Medvedev \& Loeb, 1999; Schlickeizer \& Shukla, 2003), and, perhaps, some other.

Here we discuss the scenario of generation of cosmological field by collisionless shocks during the largescale structure formation. The general prediction that shocks can generate magnetic fields was made by Moiseev \& Sagdeev (1963) and Medvedev \& Loeb (1999). Schlickeizer \& Shukla (2003) investigated the plasma physical aspects of the problem pertinent to the cosmological environment. Numerical 3D PIC simulations (Silva, et al. 2003; Nishikawa, et al. 2003; Frederiksen, et al. 2004) fully confirmed these theoretical predictions.

Proceedings of The 3rd Korean Astrophysics Workshop on Cosmic Rays and Magnetic Fields in Large Scale Structure

\section{THE MECHANISM}

In general, shocks with the Mach number greater than three, must be highly turbulent. The source and the mechanism of the turbulence is thought to be of kinetic nature, in order to prevent multi-stream motion of plasma particles. It has been shown that the Weibel instability operates at the shock front (Moiseev \& Sagdeev, 1963; Medvedev \& Loeb, 1999). This instability is driven by the anisotropy of the particle distribution function (PDF) associated with a large number of particles reflected from the shock potential.

\section{(a) Linear Regime of Field Growth and Its Saturation}

\section{i) theoretical analysis}

The instability under consideration was first predicted by Weibel (1959) for a non-relativistic plasma with an anisotropic distribution function. The simple physical interpretation provided later by Fried (1959) treated the PDF anisotropy more generally as a twostream configuration of cold plasma. Below we give a brief, qualitative description of this two-stream magnetic instability.

Let us consider, for simplicity, the dynamics of one species only (e.g., protons), whereas the other is assumed to provide global charge neutrality. ${ }^{*}$ The electrons are assumed to move along the $z$-axis with the velocities $\mathbf{v}=+\hat{z} v_{z}$ and $\mathbf{v}=-\hat{z} v_{z}$, thus forming equal particle fluxes in opposite directions (so that the net current is zero). Such a particle distribution occurs naturally near the front of a shock (moving along $z$ -

\footnotetext{
* In reality, the role of protons is more complicated. In particular, they can play a crucial role in the electron acceleration in the downstream region. We do not consider such effects in this paper; they will be studied elsewhere.
} 
direction), where the "incoming" (in the shock frame) ambient gas particles meet the "outgoing" particles reflected from the shock potential (loosely speaking, the low energy cosmic rays). Thus, the particle velocities $v_{z}$ are of order the shock velocity, $v_{z} \sim v_{\mathrm{sh}}$. The counterstreaming particles may also have some thermal spread. Since for high-Mach number shocks, $v_{\text {th }} \ll v_{\text {sh }}$ in the upstream region, we may neglect the parallel velocity spread in our consideration. The thermal spread in the transverse direction cannot be neglected, however. We parameterize the PDF anisotropy as follows:

$$
A=\frac{\epsilon_{\|}-\epsilon_{\perp}}{\epsilon_{\text {tot }}} \simeq \frac{M^{2}-1}{M^{2}+1},
$$

where $\epsilon_{\|} \propto v_{z}^{2} \simeq v_{\mathrm{sh}}^{2}$ is the energy of particle along $z$ direction, $\epsilon_{\perp} \propto\left(v_{x}^{2}+v_{y}^{2}\right) \propto v_{\text {thermal }}^{2} \simeq c_{s}^{2}$ is the thermal energy in the transverse direction, $\epsilon_{\text {tot }}=\epsilon_{\|}+\epsilon_{\perp}$ is the total energy, $c_{s}$ is the sound speed upstream and the Mach number of the shock is $M=v_{\mathrm{sh}} / c_{s}$. Clearly, for strong shocks $M \gg 1$, the anisotropy parameter is close to unity, $A \sim 1$. Next, according to the linear stability analysis technique, we add an infinitesimal magnetic field fluctuation, $\mathbf{B}=\hat{x} B_{x} \cos (k y)$. The Lorentz force, $e(\mathbf{v} \times \mathbf{B}) / c$, acts on the charged particles and deflects their trajectories, as is shown in Figure 1a. As a result, the protons moving upward and those moving downward will concentrate in spatially separated current filaments. The magnetic field of these filaments appears to increase the initial magnetic field fluctuation. The growth rate and the wavenumber of the fastest growing mode (which, in fact, sets the spatial correlation scale of the produced field) are

$$
\gamma_{B}=A \omega_{p, s}\left(v_{z} / c\right), \quad k_{B}=A \omega_{p, s} / c,
$$

where

$$
\omega_{p, s}=\left(\frac{4 \pi e^{2} n_{s}}{m_{s}}\right)^{1 / 2} \approx 1.32 \times 10^{3}\left(\frac{n_{s}}{n_{p}} \frac{m_{p}}{m_{s}}\right)^{1 / 2} \mathrm{~s}^{-1}
$$

is the plasma frequency defined for species $s$ (electrons, protons, etc.), $n_{p}$ and $m_{p}$ are the number density and the mass of the protons, respectively. (We use cgs units throughout the paper, unless stated otherwise.) Thus, the instability is indeed driven by the PDF anisotropy and should quench for the isotropic case. To put these facts in the context of cosmological shocks, we give estimates of the characteristic temporal and spatial scales. The order of magnitude estimates of the magnetic field $e$-folding time and the field correlation length at strong shocks $(M \gg 1)$ are readily obtained as

$$
\begin{aligned}
& \tau_{B} \sim 1 / \gamma_{B} \simeq 2 \times 10^{2} v_{7}^{-1} n_{-4}^{-1 / 2} \mathrm{~s}, \\
& \lambda_{B} \sim 2 \pi / k_{B} \simeq 10^{10} n_{-4}^{-1 / 2} \mathrm{~cm}
\end{aligned}
$$

for a typical IGM proton density of $n \sim 10^{-4} \mathrm{~cm}^{-3}$ and a typical shock velocity $v \sim 10^{7} \mathrm{~cm} \mathrm{~s}^{-1}$; as usual, we denote $n_{-4}=n /\left(10^{-4} \mathrm{~cm}^{-3}\right)$ and $v_{7}=v /\left(10^{7} \mathrm{~cm} \mathrm{~s}^{-1}\right)$.
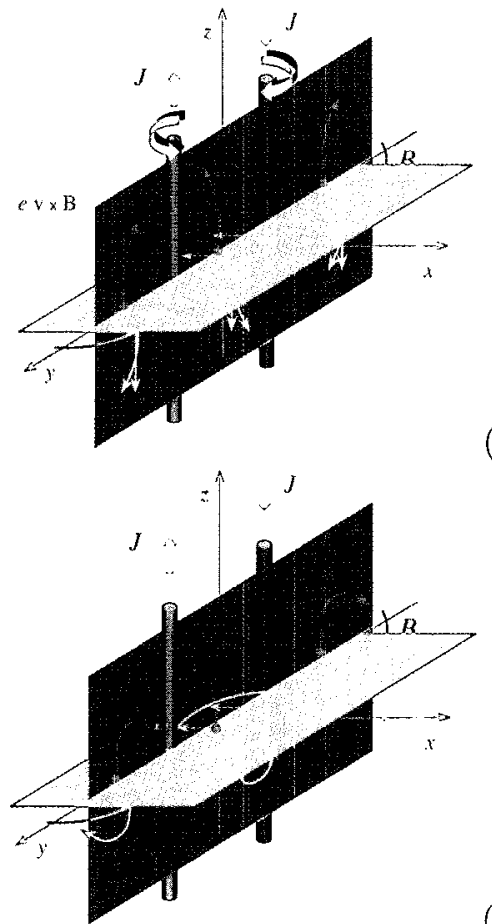

(a)

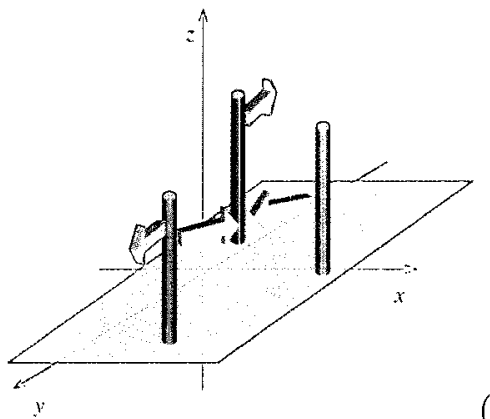

(b)

(c)

Fig. 1.- Illustration of various stages of the Weibel instability. Color coding of particles: blue - the incoming particles from the IGM, red - the particles scattered from the shock. (a) Linear regime: current filamentation; (b) saturation; (c) nonlinear regime: filament coalescence.

The Lorentz force deflection of particle orbits increases as the magnetic field perturbation grows in amplitude. The amplified magnetic field is random in the plane perpendicular to the particle motion, since it is generated from a random seed field. Thus, the Lorentz deflections result in a pitch angle scattering, which makes the bulk of the PDF isotropic. If one starts from a strong anisotropy, so that the thermal spread is much smaller than the particle bulk velocity, most of the particles will eventually isotropize and the thermal energy associated with their random motions will be equal to their initial directed kinetic energy. This final state will bring the instability to saturation.

The saturation level of the magnetic field may readily be estimated as follows. First of all, note that the instability is due to the free streaming of particles. As the magnitude of the magnetic field grows, the trans- 
verse deflection of particles gets stronger, and their free streaming across the field lines is suppressed, see Figure 1b. The typical curvature scale for the deflections is the Larmor radius,

$$
\rho_{L}=v_{\perp B} / \omega_{c, s}
$$

where $v_{\perp B}$ is the particle velocity transverse to the direction of the local magnetic field and

$$
\omega_{c, s}=\frac{e B}{m_{s} c} \approx 9.58 \times 10^{3} B\left(m_{p} / m_{s}\right) \mathrm{s}^{-1}
$$

is the cyclotron (Larmor) frequency of species $s$. On scales larger than $\rho_{L}$, particles can. only move along field lines. Hence, when the growing magnetic fields become such that $k_{B} \rho_{L} \sim 1$, the particles are magnetically trapped and can no longer amplify the field. Assuming an isotropic particle distribution at saturation $\left(v_{\perp B} \sim v_{\mathrm{sh}}\right)$, this condition can be re-written as

$$
\epsilon_{B}=\frac{B^{2} / 8 \pi}{m_{s} n_{s} v_{\mathrm{sh}}^{2} / 2} \simeq A^{2} .
$$

For strong shocks $(M \gg 1, A \sim 1)$, this corresponds to the magnetic energy density close to equipartition with the thermal energy of particles downstream the shock.

\section{ii) 3D PIC simulations of the instability}

The dynamics of the Weibel instability has recently been simulated by our group (Silva, et al. 2003) as well as by several other research groups using 3D plasma kinetic codes (Nishikawa et al., 2003; Frederiksen et al., 2004). We examined the instability, which occurs in a collision of two inter-penetrating unmagnetized electron-positron clouds with zero net charge. This is the simplest model for the formation region of a shock front, as well as a classic scenario unstable to electromagnetic and/or electrostatic plasma instabilities.

The relativistic electromagnetic 3D PIC code OSIRIS (Fonseca, et al., 2002) was used. The simulations were performed in a simulation cube of size $256 \times 256 \times 100$ grid points, ten grid points correspond to one plasma skin depth $c / \omega_{p, e}$. We had more than $10^{8}$ particles in the simulation box. Periodic boundary conditions were imposed. The initial state is spatially homogeneous with two identical groups of particles moving with some velocity $\pm v_{z}$. The particles in both groups have a small thermal velocity $v_{\text {th }} \simeq v_{z} / 6$. The system has no net charge and no net current, and initially the electric and magnetic fields are set to zero.

The results of the simulations are shown in Figure 2 and 3 . Figure 2 shows the temporal evolution of the magnetic equipartition parameter $\epsilon_{B}$. In Figure 3 the three-dimensional structure of the magnetic fields and currents are shown at two different times: (a) during linear regime, at $t \simeq 13 \omega_{p, e}^{-1}$, and (b) just after the saturation, at $t \simeq 20 \omega_{p, e}^{-1}$. The left panels show the structure of magnetic field lines and the right panels

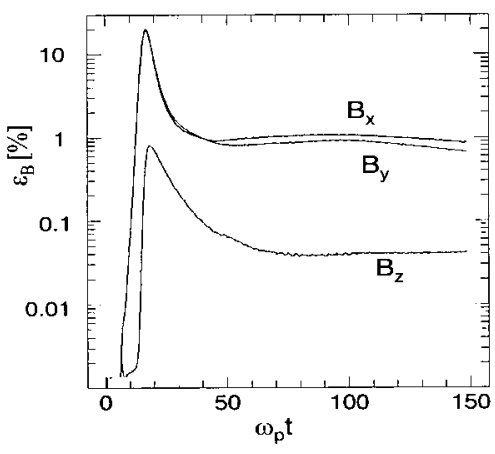

Fig. 2. - The temporal evolution of the magnetic field energy density normalized by the initial kinetic energy of the particles.

show the number density of particles (blue are moving downward, red are moving upward). We see that during the linear stage of the instability $\left(\omega_{p, e} t \lesssim 15\right)$ there is exponential generation of a magnetic field, which predominantly lies in the plane of the shock $(x-y$ plane), i.e., perpendicular to the direction of motion of the plasma clouds. The produced magnetic field is highly inhomogeneous, with the characteristic correlation scale comparable to the plasma skin depth length $c / \omega_{p, e}$. It is also seen that the magnetic field generation is associated with the separation of the particle streams in spatially distinct regions and the formation of straight current filaments.

Saturation of the instability occurs at time $t \sim$ $15 \omega_{p, e}^{-1}$, which is indicated by the peak of $\epsilon_{B}$ in Figure 2. At this moment, most of the particles are randomized over the pitch angle by the Lorentz deflections. Thus the PDF anisotropy, which is the free energy source for the instability is removed. At the time of saturation, the magnetic field energy density reaches its maximum $\sim 20 \%$.

At longer times, one sees the substantial decrease in $\epsilon_{B}$, during the initial stage of the nonlinear regime in which current filaments begin to interact with each other, forcing like currents to approach each other and merge. During this phase, initially randomly oriented filaments cross each other to form a more organized, large-scale quasi-regular pattern, hence much current and B-field is annihilated. At later times, $t \gtrsim 35 \omega_{p, e}^{-1}$, the filament coalescence continues, as is indicated by the increase of the filament sizes. Note that the spatial distribution of currents is now quite regular, so that filaments with opposite polarity no longer cross each other, but simply interchange, staying always far away. The total magnetic field energy is $\epsilon_{B} \sim 0.25 \%$ and does not change any more. Note also that the residual magnetic field is highly inhomogeneous, seen as a collection of magnetic field filaments or "bubbles". The amplitude of the field in the bubbles is close to equipartition. Therefore, the overall decrease of the magnetic field energy is mostly associated with the decreasing filling factor of the field.

We discuss the nonlinear field evolution in the next 

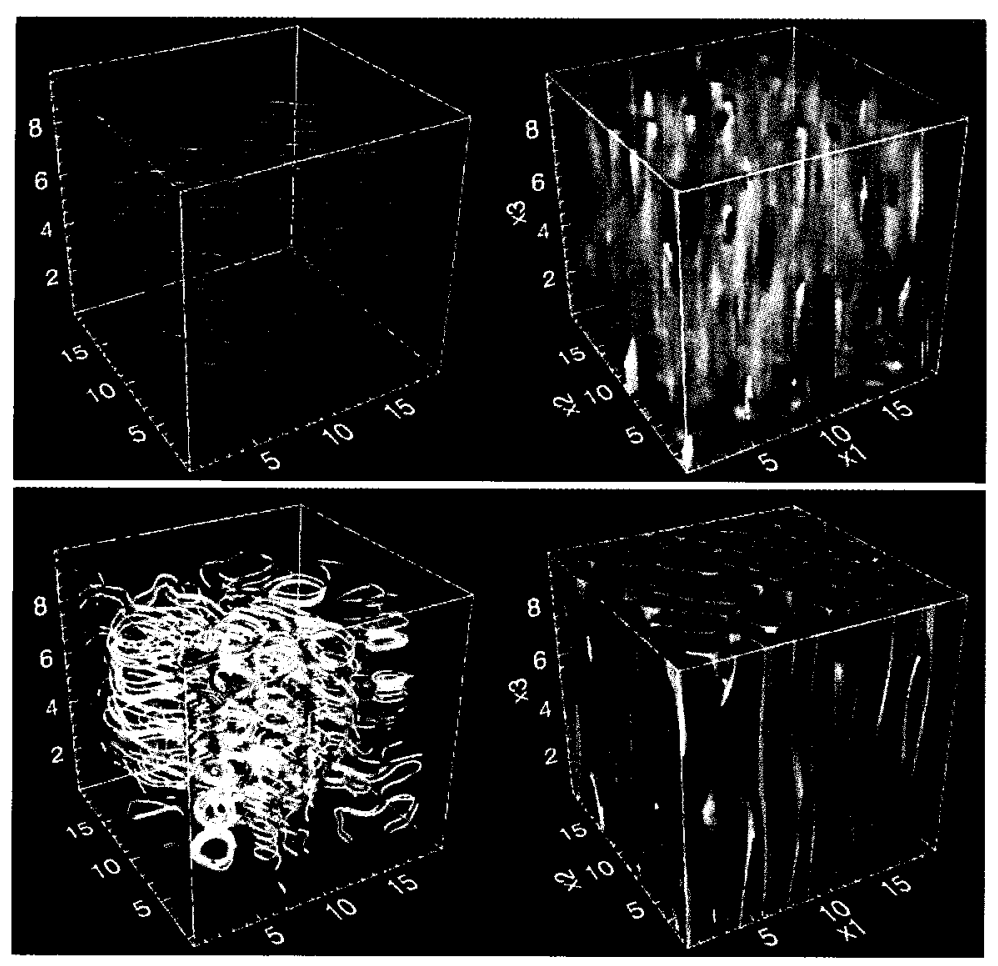

Fig. 3.- The 3D structure of the magnetic fields and currents from the simulations at two different times: (a) during linear regime, at $t \simeq 13 \omega_{p, e}^{-1}$, and (b) just after the saturation, at $t \simeq 20 \omega_{p, e}^{-1}$. The left panels show the structure of magnetic field lines and the right panels show the number density of particles (blue are moving downward, red are moving upward).

section in great details.

\section{(b) Nonlinear Regime: Long-Time Field Evo- lution}

Whereas there is no doubt that magnetic field is generated at shocks, there was a great concern about the life-time of these fields, i.e., whether they can populate a substantial volume downstream. The concern arises from the fact that the fields produced are tangled on a very small scale,

$$
\lambda_{B} \sim 2 \pi c / \omega_{p, p} \simeq 10^{10} \mathrm{~cm}
$$

for a typical IGM particle density of $n \sim 10^{-4} \mathrm{~cm}^{-3}$. Therefore, it is possible that the extremely short spatial scales, i.e., sharp field gradients, must be rapidly destroyed by dissipation, on a plasma time-scale of $\tau_{B} \sim$ $10^{2}$ s. Should this happen, the fields would occupy only a very narrow region near the shock front and, thus, cannot be the source of large-scale cosmological fields. Therefore, understanding of the long-term evolution of the Weibel magnetic fields is of great importance. Recently, we performed such an analysis using both theoretical and numerical methods (Medvedev, et al. 2005) and demonstrated that the field should not decay to zero as $t \rightarrow \infty$. We briefly outline the analysis and the results here.

\section{i) theoretical analysis}

Our 3D PIC numerical simulations described above demonstrate that the generated magnetic fields are associated with a quasi-two-dimensional distribution of current filaments (Silva, et al. 2003). Hence we suggest the following toy model, see Figure 1c.

We consider straight one-dimensional current filaments oriented in the vertical, $z$-direction. Initially, all filaments are identical: the initial diameter of them is $D_{0}$, their initial mass per unit length is $\mu_{0} \simeq$ $m n\left(\pi D_{0}^{2} / 4\right)$, where $m$ is the mass of plasma particles (e.g., electrons) and $n$ is their number density. Each filament carries current $I_{0}$ in either positive or negative $\hat{z}$-direction. The net current in the system is set to zero, i.e., there are equal numbers of positive and negative current filaments. We also assume that the initial separation (the distance between the centers) of the filaments $d_{0}$ is comparable to their size, $d_{0} \simeq 2 D_{0}$. Finally, no external homogeneous magnetic field is present in the system.

Initially, the filaments are at rest and their positions in space are random. This configuration is unstable because opposite currents repel each other, whereas like currents are attracted to each other and tend to coalesce and form larger current filaments. The characteristic scale of the magnetic field will accordingly increase with time. We study this process quantitatively using the toy model of two interacting filaments.

The magnetic field produced by a straight filament 
is $B_{0}(r)=2 I_{0} /(\mathrm{cr})$, where $r$ is the cylindrical radius. The force per unit length acting on the second filament is $d F / d l=-B_{0} I_{0} / c$. Since $d F / d l=\mu \ddot{x}$, where $x$ is the position in the center of mass frame and "overdot" denotes time derivative, we write the equation of motion as follows:

$$
\ddot{x}=-\frac{2 I_{0}^{2}}{c^{2} \mu_{0}} \frac{1}{x},
$$

where we used that $r=2 x$ and the reduced mass $\mu_{r}=\mu_{0} / 2$. We define the coalescence time as the time required for the filaments, which are initially at rest, to cross the distance between them and "touch" each other, which happens when the distance between their centers becomes equal to $D_{0}$, i.e., when $x=D_{0} / 2$. The coalescence time, as it is defined above, is independent of the details of the merging process itself, which involves rather complicated dynamics associated with the redistribution of currents. Quite obviously, the interaction between the filaments is the weakest at large distances $x \sim x_{0} \sim d_{0} / 2$. Hence, the coalescence rate is limited by the filament motions at the largest scales. The coalescence time can be readily estimated from Eq. (10), assuming that $x \sim x_{0} \sim d_{0} / 2$ and $\ddot{x} \sim\left(d_{0} / 2\right) \tau_{0}^{-2}$, as follows:

$$
\tau_{0, N R} \sim\left(D_{0}^{2} c^{2} \mu_{0} /\left(2 I_{0}^{2}\right)\right)^{1 / 2}
$$

The above estimate is valid as long as the motion is non-relativistic. The maximum velocity of a filament is at the time of coalescence, $x=D_{0} / 2$ :

$$
v_{m 0} \sim D_{0} / 2 \tau_{0} \sim I_{0} /\left(c \sqrt{2 \mu_{0}}\right) .
$$

It must always be much smaller than the speed of light.

If the motion of a filament during the merger becomes relativistic, i.e., $v_{m 0}$ becomes comparable to $c$ the separation cannot decrease faster than as $t(x) \simeq$ $x / c$. Therefore, the coalescence time will be

$$
\tau_{0, R} \simeq\left(d_{0} / 2\right) / c=D_{0} / c .
$$

The filament coalescence is a hierarchical process. Indeed, suppose that initially the system contains $N_{0}$ current filaments, with an average separation $d_{0} \sim$ $2 D_{0}$. Each of the filaments carries current $I_{0}$, its diameter is $D_{0}$ and its mass per unit length is $\mu_{0}$. For simplicity, we assume that filaments coalesce pairwise.

Having the original "zeroth generation" of filaments merged (the process takes about $\tau_{0, N R}$ or $\tau_{0, R}$ to complete), the system will now contain $N_{0} / 2$ of "first generation" filaments. Each of these filaments carries current $I_{1}=2 I_{0}$, has mass per unit length $\mu_{1}=2 \mu_{0}$, and the separation between them is $d_{1}=\sqrt{2} d_{0}$ (because the two dimensional number density of filaments decreased by 2). Since $\mu \propto D^{2}$, the filament size also increases as $D_{1}=\sqrt{2} D_{0}$. Remarkably, this new configuration is identical to the initial one, but with the re-scaled parameters. Hence, the coalescence process is self-similar.
The produced first generation filaments will be interacting with each other and merge again to yield the second generation. The coalescence process will then continue in a self-similar way. Note that the coalescence times at each stage are not necessarily the same. Taking into account that at the $k$-th merger level, i.e., after $k$ pairwise mergers: $I_{k}=2^{k} I_{0}, \mu_{k}=2^{k} \mu_{0}, D_{k}=$ $2^{k / 2} D_{0}, d_{k} \sim D_{k} / 2$, we obtain

$$
\tau_{k, N R}=\tau_{0, N R}, \quad \tau_{k, R}=2^{k / 2} \tau_{0, R}
$$

Since the coalescence time is independent of $k$ while the filaments are non-relativistic, whereas the distance between them increases, the typical velocities of the merging filaments grow with time and, will approach $c$, as $v_{m, k}=2^{k / 2} v_{m 0}$. The transition from the nonrelativistic regime to the relativistic one occurs after about $k_{*}$ mergers:

$$
k_{*}=2 \log _{2}\left(c / v_{m 0}\right),
$$

where $v_{m 0}$ is set by the initial state of the system, Eq. (12).

Finally, it is instructive to present the evolution of the parameters as a function of physical time, $t$, rather than the merger level, $k$. Apparently, it takes $t=\sum_{k^{\prime}=0}^{k} \tau_{k^{\prime}}$ to complete $k$ mergers, where $\tau_{k}$ is given by Eq. (14). Thus, for the non-relativistic and relativistic cases respectively, we have: $k=t / \tau_{0, N R}$ and $k \simeq 2 \log _{2}\left[\left(t / \tau_{0, R}\right)\right]$. Thus, the magnetic field correlation length increases as a function of time as

$$
\lambda_{B}(t)=D_{0} 2^{t /\left(2 \tau_{0, N R}\right)}, \quad \lambda_{B}(t) \simeq c t,
$$

in the non-relativistic and relativistic regimes, respectively. Note that the last expression is an approximation at large times $t \gg \tau_{0}, R$, i.e., at large $k \gg 1$.

We now represent the main results in terms of the parameters of a shock and IGM. First, the initial separation between the filaments, $D_{0}$, must be comparable to the characteristic correlation length of the magnetic field produced by the instability. This length at the onset of the instability is, in turn, set by the wavenumber of the fastest growing mode: $\lambda_{s} \simeq c / \omega_{p, s}$. However, we cannot set this scale $\lambda_{s}$ as $D_{0}$ because, as indicated by $3 \mathrm{D} \mathrm{PIC} e^{-} e^{+}$simulations (Silva, et al. 2003), $\epsilon_{B}$ is not constant in time at the beginning of the nonlinear filament interaction (at $t \sim 10 \omega_{p, s}^{-1}$ ). Hence the above analysis is not applicable in such a regime. In fact, it takes few plasma times, $\omega_{p, s}^{-1}$, in their simulations for $\epsilon_{B}$ to attain its asymptotic value. The field correlation scale at this moment $\left(t \sim 15 \omega_{p, s}^{-1}\right)$ is somewhat larger than $\lambda_{s}$. We include this uncertainty via the parameter $\eta>1$ as follows:

$$
D_{0} \sim \eta\left(c / \omega_{p, s}\right)
$$

Second, using $\oint \mathbf{B} \cdot d \mathbf{l}=(4 \pi / c) I$, we express the current $I_{0}$ in terms of the equipartition parameter, $\epsilon_{B}$, as $I_{0} \sim$ 

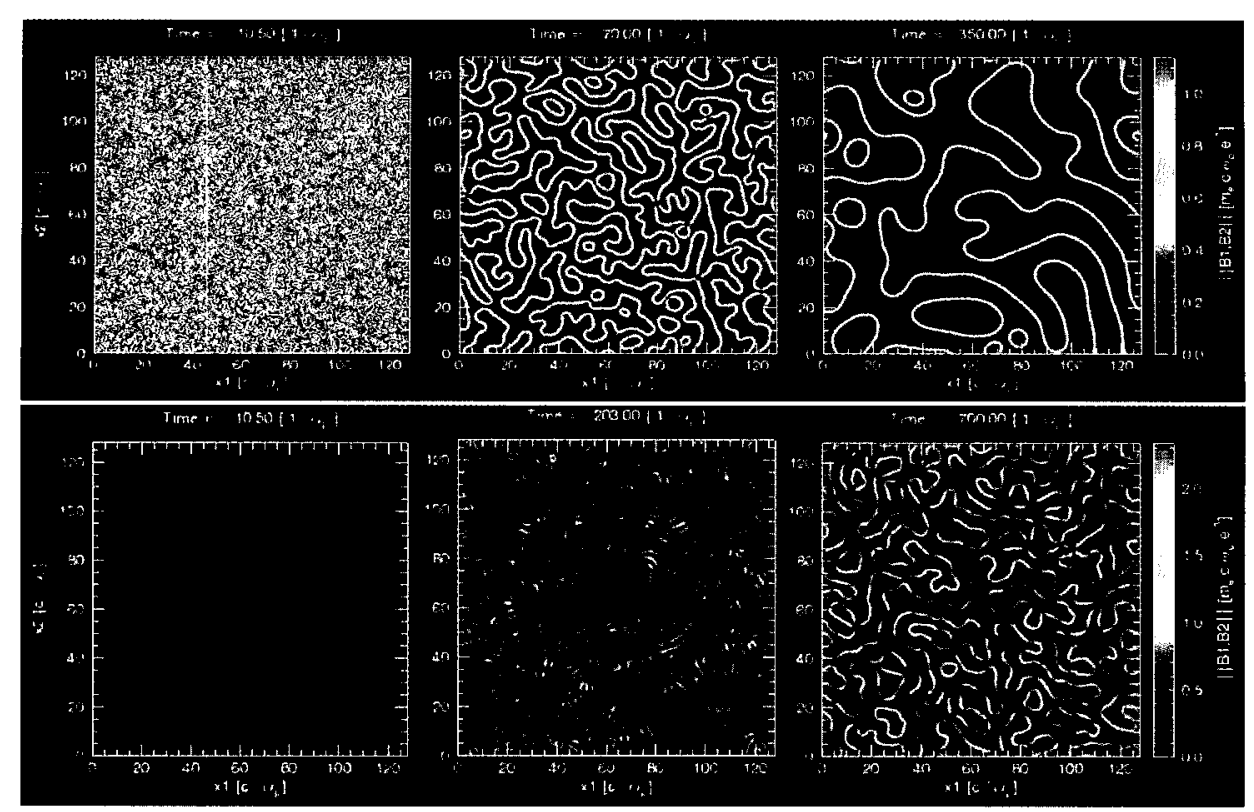

Fig. 4. - The 2D structure of the magnetic fields in $e^{-} e^{+}$(top panel) and $e^{-} p$ with $m_{p} / m_{e}=1860$ (bottom panel) runs at various times. The change of the field correlation length with time is clearly seen. The growth of this length is substantially slower and the magnetic field filling factor is respectively larger in the electron-proton run.

$\eta \sqrt{\epsilon_{B}}\left(m_{s} v_{\mathrm{sh}} c^{2} / e\right)$. Third, the mass per unit length of a filament is $\mu_{0} \sim m_{s} n_{s} D_{0}^{2}$.

The temporal evolution of the field correlation scale is determined by Eq. (16), where $\tau_{0, N R}$ is given by (11). The coalescence time may be written as

$$
\tau_{0, N R} \sim=\frac{\eta\left(c / v_{\mathrm{sh}}\right)}{\sqrt{\epsilon_{B}} \omega_{p, s}} \sim 10^{5} \omega_{p, s}^{-1}
$$

Hereafter we assumed the typical values: $\epsilon_{B} \sim 10^{-3}$ and $\eta \sim 1$. The maximum merger velocity $v_{m 0}$, Eq. (12), in terms of the speed of light is $v_{m 0} / c \sim \sqrt{\epsilon_{B}} \sim$ 0.03. The transition from the non-relativistic to relativistic coalescence regime occurs after $k_{*}$ mergers, given by Eq. (15). i.e., at the time

$$
t_{*}=2 \log _{2}\left(c / v_{0 m}\right) \tau_{0, N R} \sim 10 \tau_{0, N R} .
$$

\section{ii) high-resolution 2D simulations}

We now compare our theoretical predictions with the results of particle-in-cell numerical simulations, performed using code OSIRIS. We have performed 2D simulations $\left(1280 \times 1280\right.$ cells, $128.0 \times 128.0\left(c / \omega_{p, e}\right)^{2}, 9$ particles/(cell $\times$ species), 4 species) of the collision of electrically neutral clouds (electron-positron $-e^{-} e^{+}$, and electron-proton $-e^{-} p$ ) moving in the $\hat{z}$ direction, across the $x y$ simulation plane, with parameters similar to those in (Silva, et al. 2003). In order to save on computation time and trace a substantial period of field evolution, we set the particles bulk velocity to $v_{\text {sh }} \simeq v \simeq 0.6 c$. Relativistic effects do not play any significant role because the corresponding Lorentz factor is $\gamma_{\mathrm{sh}} \sim 1.17$.
Figure 4 shows the 2D structure of the magnetic fields in $e^{-} e^{+}$and $e^{-} p$ with $m_{p} / m_{e}=1836$ runs at various times. The change of the field correlation length with time is clearly seen. The growth of this length is substantially slower and the magnetic field filling factor is respectively larger in the electron-proton run. The temporal evolution of $\lambda_{B}$ as measured in the simulations is shown in Figure 5. Both a non-power-law non-

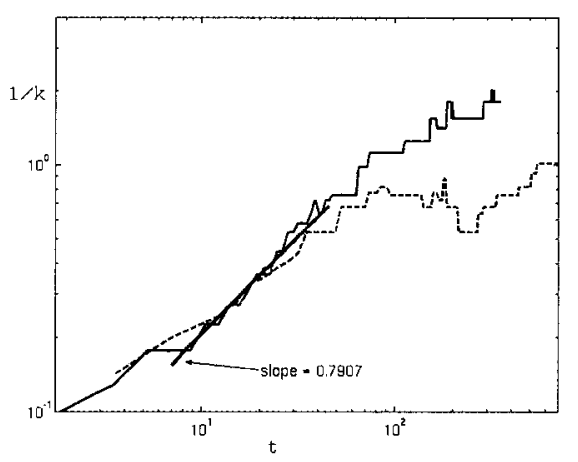

Fig. 5.- Temporal evolution of the field correlation length in electron-positron (solid line) and electron-proton (dashed line) runs. In the range $7 / \omega_{p, e}<t<50 / \omega_{p, e}, \lambda_{B} \propto t^{\alpha}$ with

rẽätivistic regime (until $t \approx 10-20 / \omega_{p, e}$ ) and a powerlaw regime are clearly seen. The power-law fits yield $\lambda_{B}(t) \propto t^{\alpha}$ with $\alpha \approx 0.8$. Note also that the second power-law segment with the same index is present at $t \gtrsim 100 / \omega_{p, e}$ in $e^{-} p$ run, indicating proton filament coalescences. A similar behavior was also observed in $3 \mathrm{D}$ simulations, but the significantly larger simulation planes presented here allow for improved statistics. At late times $t \gtrsim 100 / \omega_{p, e}$, the evolution of $\lambda_{B}$ rolls off 


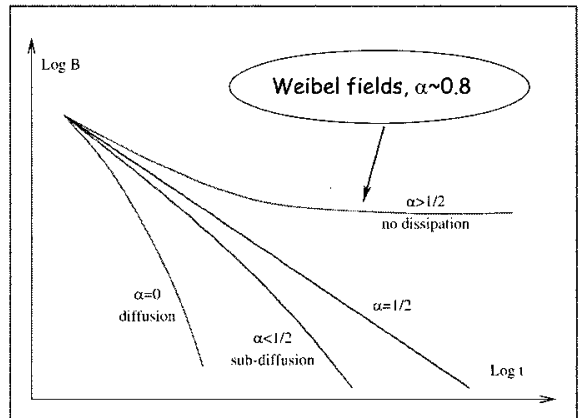

Fig. 6.- Temporal evolution of the field strength for different $\alpha$ 's.

and is slower when the number of filaments in the simulation box becomes relatively small.

Now we can answer the question: Why the generated magnetic fields do not rapidly decay back to zero as soon as the instability shuts off? The answer is: The produced fields and the corresponding currents self-organize and form a quasi-two-dimensional distribution. A typical magnetic field gradient scale grows with time very rapidly, with approximately the light crossing time $\propto t^{0.8}$; whereas the particle diffusion is a substantially slower process. Hence, diffusive dissipation is drastically reduced. To illustrate this, we consider the field diffusion equation

$$
\partial_{t} B=-\kappa \partial_{x x}^{2} B
$$

with the dissipation coefficient, $\kappa$, being constant, for simplicity. Approximating the spatial derivative as $\partial_{x} \sim \lambda_{B}(t)^{-1} \sim \lambda_{0}^{-1}\left(t / t_{0}\right)^{-\alpha}$, where $\lambda_{0}$ and $t_{0}$ are constants and $\alpha>1 / 2$, we obtain the scaling of $B$ with time as

$$
\begin{aligned}
B(t) & \propto \exp \left(-\int \lambda_{B}^{-2}(t) d t\right) \\
& \propto \begin{cases}\exp \left(-t^{1-2 \alpha}\right), & \alpha<1 / 2, \\
t^{\delta}, & \alpha=1 / 2, \\
\exp \left(t^{1-2 \alpha}-t_{0}^{1-2 \alpha}\right), & \alpha>1 / 2,\end{cases}
\end{aligned}
$$

where $t_{0}$ and $\delta$ are some constants depending on $\kappa, \lambda_{0}$ and other parameters. These relations are illustrated in Figure 6.

We also note that in some respect, the field scale growth is analogous to the inverse cascade in twodimensional magnetohydrodynamic (MHD) turbulence (see, e.g., Biskamp \& Bremer, 1994). The crucial difference is, however, the entirely kinetic nature of the process; at such small scales $\sim c / \omega_{p}$ the MHD approximation is completely inapplicable.

\section{REALISTIC 3D SIMULATIONS OF A SHOCK}

Simulations described above were aimed at the study of the linear and nonlinear dynamics of the counterstreaming instability itself. It is worthwhile, however,
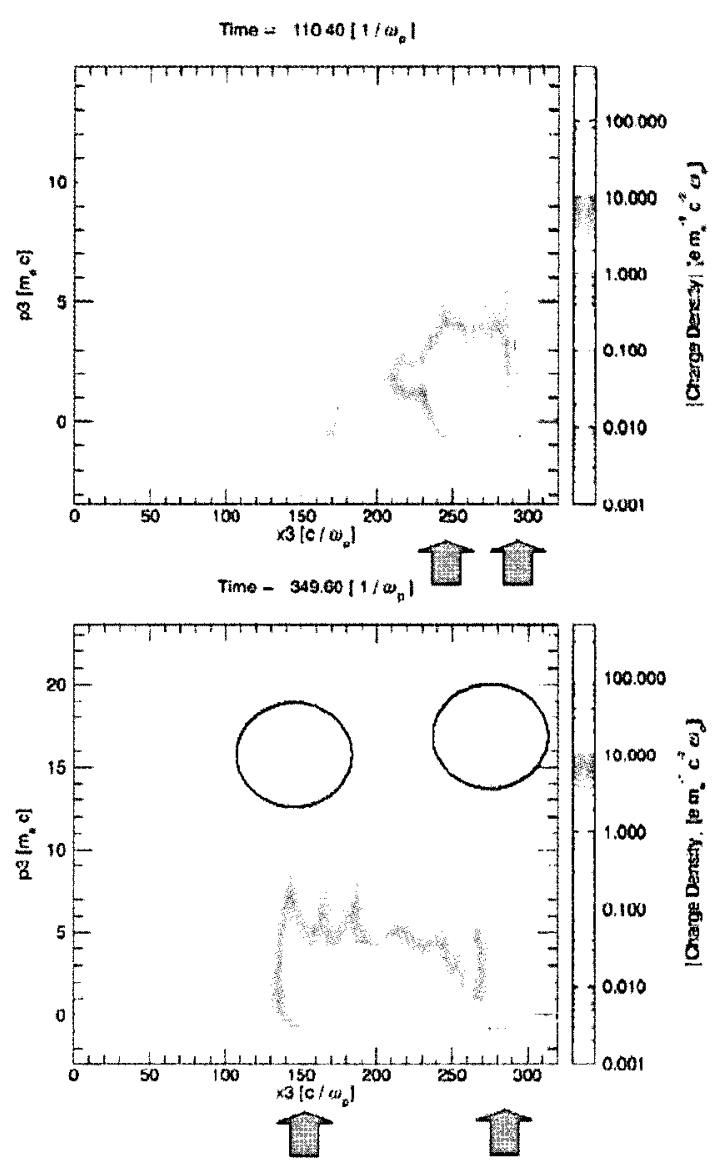

Fig. 7.- Parallel momentum of IGM particles in the 3D shock simulations at two different times. The formation and kinematics of the forward and reverse shocks are clearly seen (their positions are marked by arrows). The simulations are done in the frame co-moving with the forward shock (propagating to the right in the lab frame).

to perform a realistic simulation of the formation of collisionless shock, without the assumptions of initial spatial inhomogeneity and periodic boundary conditions. Such a simulation was performed by our group using code OSIRIS. The results of this simulation are shown

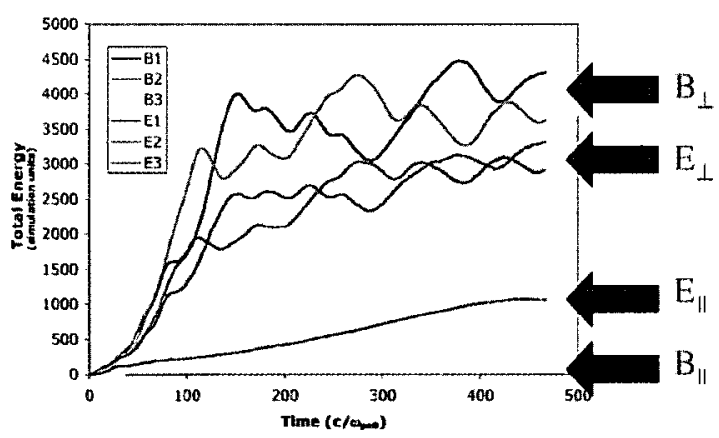

Fig. 8.- Magnetic and electric fields averaged over the simulation box in 3D shock simulations as a function of time. The generation of predominantly transverse, subequipartition magnetic fields is observed. 
in Figures 7 and 8, the former representing the parallel momentum of the IGM particles, the latter shows the volume-averaged magnetic and electric field. The simulation parameters correspond to the strong shock with the Mach number $M \simeq 6$, while the artificially large shock velocity, $v_{\text {sh }} \simeq 0.5 c$, was chosen in order to save on the computational time (note, from Eq.18, that the coalescence time is inversely proportional to $\left.v_{\mathrm{sh}}\right)$. Relativistic effects play a negligible role in this run. We have also chosen that the electrons and the protons have the same thermal velocities, not temperatures, because Coulomb collisions are very rare.

In contrast to the theoretical predictions of Schlickeizer \& Shukla (2003), who argued that only shock with Mach number greater than 43 are capable of producing magnetic fields, we demonstrate that $M \simeq 6$ shock efficiently generates a sub-equipartition field. The reason for the disagreement is that Schlickeizer \& Shukla (2003) assumed in their analysis that the electrons and the protons in the inter-penetrating streams have the same energy (temperature). While this assumption is valid for the IGM particles, it fails for the particles reflected from the shock: Coulomb collisions are very inefficient in such tenuous plasmas and equipartition between the electrons and protons cannot establish at the shock front. Instead, the velocities of the reflected particles are the same for the protons and electrons and are comparable to the shock velocity.

\section{SUMMARY OF THE RESULTS}

We demonstrated that magnetic fields are naturally generated in collisionless cosmological shocks. These magnetic fields have random orientation and predominantly lie in the plane of the shock. The correlation length (in the shock plane) of the fields is very small:

$$
\lambda_{B} \sim 2 \pi c / \omega_{p} \simeq 10^{10} n_{\mathrm{IGM},-4}^{-1 / 2} \mathrm{~cm}
$$

The magnetic field generation occurs very fast, with the $e$-folding time

$$
\tau_{B} \sim\left(c / v_{\mathrm{sh}}\right) \omega_{p}^{-1} \simeq 2 \times 10^{2} v_{\mathrm{sh}, 7}^{-1} n_{\mathrm{IGM},-4}^{-1 / 2} \mathrm{~s} .
$$

It is these fields that pitch-angle scatter the particles and introduce effective collisions in the otherwise collisionless system. Since it takes $N \sim$ few $\times 10 e$-foldings to produce strong fields, the shock thickness is, thus, determined as

$$
\Delta_{\mathrm{sh}} \sim N \tau_{B} v_{\mathrm{sh}} \sim N \lambda_{B} \sim \text { few } \times 10^{11} n_{\mathrm{IGM},-4}^{-1 / 2} \mathrm{~cm} .
$$

At the shock front, the magnetic field energy density reaches about a quarter of the initial kinetic energy density, $\epsilon_{B} \sim 0.25$, and, hence, constitute a similar fraction of the thermal energy density of the shocked gas.

Farer downstream, at distances much larger that $\Delta_{\text {sh }}$ from the front, the magnetic field strength drops and asymptotes at

$$
\epsilon_{B}=\frac{B^{2} / 8 \pi}{m_{p} n_{\mathrm{IGM}} v_{\mathrm{sh}}^{2} / 2} \sim \frac{B^{2}}{8 \pi p_{\mathrm{sh}}} \sim 10^{-3} \ldots 10^{-4},
$$

where $p_{\text {sh }}$ is the gas pressure behind the shock. the actual number depends on complicated nonlinear dynamics of the currents in the downstream region. This value of the equipartition parameter corresponds to the magnetic field strength of order

$$
B \sim 10^{-8} \epsilon_{B,-3}^{1 / 2} v_{\mathrm{sh}, 7} n_{\mathrm{IGM},-4}^{1 / 2} \text { gauss. }
$$

The magnetic field correlation length increases rapidly with time:

$$
\lambda_{B}(t) \sim c t
$$

which makes these fields to be weakly coupled to dissipation. Hence the shock-generated magnetic fields produced by large-scale structure shocks can populate cosmologically large volumes.

\section{ACKNOWLEDGEMENTS}

The authors are grateful to Mark Kamionkowsky for a discussion. One of the authors (MM) has been supported by NASA grant NNG-04GM41G and DoE grant DE-FG02-04ER54790. The work of some of the authors (MF, RAF, and LOS) is partially supported by FCT (Portugal).

\section{REFERENCES}

Biermann, P. 1950, Z. Naturf. A., 5, 65

Biskamp, D., \& Bremer, U. 1994, Phys. Rev. Lett., 72, 3819

Field, G. B., \& Carroll, S. M. 2000, Phys. Rev. D, 62, 103008

Fonseca, R. A., et al. 2002, Lecture Notes in Computer Science 2329, III-342 (Heidelberg: Springer-Verlag)

Frederiksen, J. T., Hededal, C. B., Haugbølle, T., \& Nordlund, A. A. 2004, ApJL, 608, L13

Gasperini, M., Giovannini, M., \& Veneziano, G. 1995, Phys. Rev. Lett., 75, 3769

Gnedin, N. Y., Ferrara, A., \& Zweibel, E. G. 2000, ApJ, 539,505

Harrison, E. R. 1970, MNRAS, 147, 279

Medvedev, M. V., \& Loeb, A. 1999, ApJ, 526, 697

Medvedev, M. V., Fiore, M., Fonseca, R. A., Silva, L. A., \& Mori, W. B. 2005, ApJ, submitted

Moiseev, S. S., \& Sagdeev, R. Z. 1963, J. Nucl. Energy C, 5,43

Nishikawa, K.-I., Hardee, P., Richardson, G., Preece, R., Sol, H., \& Fishman, G. J. 2003, ApJ, 595, 555

Quashnock, J. M., Loeb, A., \& Spergel, D. N. 1989, ApJ, 344, L49

Ratra, B. 1992, ApJ, 391, L1

Sicotte, H. 1997, MNRAS, 287, 1 
Sigl, G. 2002, Phys. Rev. D, 66, 123002

Sigl, G., Olinto, A. V., \& Jedamnik, K. 1997, Phys. Rev. D, 55,4582

Silva, L. O., Fonseca, R. A., Tonge, J. W., Dawson, J. M., Mori, W. B., \& Medvedev, M. V. 2003, ApJ, 596, L121

Vachaspati, V. 1991, Phys. Lett. B, 265, 258 